Bull. Austral. Math. Soc.

$90 \mathrm{c} 31,90 \mathrm{c} 30$

VOL. 44 (1991) [501-509]

\title{
DUALITY IN NONCONVEX VECTOR MINIMUM PROBLEMS
}

\author{
T.R. Gulati and Nadia Talaat
}

A nonlinear vector minimum problem is considered. Duality theorems are proved for Mond-Weir type dual and their application to a certain nonlinear fractional vector minimum problem is discussed.

\section{INTRODUCTION}

Consider the vector minimum problem:

$$
\text { minimise } f(x) \text { subject to } g(x) \leqq 0
$$

where $f: R^{n} \rightarrow R^{k}$ and $g: R^{n} \rightarrow R^{m}$ are differentiable functions. Wolfe and MondWeir type duality for $(P)$ has been discussed in several papers $[1,7,8,9,10]$ using different concepts of optimality, namely: weak efficient (or weak minimum), efficient (or nondominated or noninferior or Pareto optimal) and properly efficient solutions.

Weir [8] proved weak and strong duality theorems for Mond-Weir [5] type dual of $(P)$. In the strong duality theorem he obtained an efficient solution of the dual from a properly efficient solution of the primal problem. Bector et al [1] also discussed a similar result under stronger convexity assumptions. The duality results in Singh [7] and Weir and Mond [10] are for efficient and weak efficient solutions respectively. The converse duality theorems in $[1,7]$ are proved using Kuhn-Tucker type necessary conditions of Singh [6] and therefore need a constraint qualification.

In the present paper we also discuss duality results for Mond-Weir type dual of $(P)$. Our results are different than those in $[1,7,8,10]$. The strong duality theorem provides a properly efficient solution of the dual while in the converse duality theorem a weak efficient solution of the dual gives a properly efficient solution of the primal problem. Moreover, the converse duality theorem is proved using Fritz John necessary conditions [2] which do not need a constraint qualification.

Received 22 December 1990

The second author would like to thank the Government of India for providing her financial support under Indo-ARE Cultural Exchange Programme.

Copyright Clearance Centre, Inc. Serial-fee code: 0004-9729/91 SA2.00+0.00. 


\section{Preliminaries}

The following convention of vectors in $R^{n}$ will be followed throughout this paper: $x \leqq y \Leftrightarrow x_{i} \leqq y_{i}, i=1,2, \ldots, n ; x \leqq y \Leftrightarrow x \leqq y, x \neq y ; x<y \Leftrightarrow x_{i}<y_{i}$, $i=1,2, \ldots, n . \nabla g(x)$ will denote the $m \times n$ Jacobian matrix of $g$ at $x$, the index set $K=\{1,2, \ldots, k\}$ and $K_{i}=K-\{i\}$. For other notations and definitions we refer to Mangasarian [4].

Geoffrion [3] introduced the following scalar parametric problem:

$$
\text { minimise } \lambda^{T} f(x) \text { subject to } g(x) \leqq 0
$$

and related its optimal solution to a properly efficient solution of $(P)$ as follows:

Lemma 1. Let $\lambda>0$ be fixed. If $\bar{x}$ is an optimal solution of $\left(P_{\lambda}\right)$, then $\bar{x}$ is a properly efficient solution of $(P)$.

The Comprehensive Theorem in Geoffrion [3] includes the following necessary and sufficient conditions for problem $(P)$ :

Lemma 2. (Kuhn-Tucker type necessary conditions). Let $\bar{x}$ be a properly efficient solution of problem $(P)$ and let $g$ satisfy the Kuhn-Tucker constraint qualification at $\bar{x}$. Then there exist $\bar{\lambda} \in R^{k}, \bar{\mu} \in R^{m}$ such that

$$
\begin{aligned}
\bar{\lambda}^{T} \nabla f(\bar{x})+\bar{\mu}^{T} \nabla g(\bar{x}) & =0 \\
\bar{\mu}^{T} g(\bar{x}) & =0 \\
\bar{\lambda}>0, \bar{\mu} & \geqq 0 .
\end{aligned}
$$

Lemma 3. (Kuhn-Tucker type sufficient conditions). Let $f$ and $g$ be convex. If there exist $\bar{\lambda} \in R^{k}$ and $\bar{\mu} \in R^{m}$ such that

$$
\begin{aligned}
\bar{\lambda}^{T} \nabla f(\bar{x})+\bar{\mu}^{T} \nabla g(\bar{x}) & =0 \\
\bar{\mu}^{T} g(\bar{x}) & =0 \\
\bar{\lambda}>0, \bar{\mu} & \geqq 0,
\end{aligned}
$$

then $\bar{x}$ is a properly efficient solution of $(P)$.

\section{DuAlity}

In relation to $(P)$ we consider the following Mond-Weir type dual:

$$
\begin{aligned}
& \operatorname{maximise} f(y) \\
& \text { subject to } \quad \lambda^{T} \nabla f(y)+\mu^{T} \nabla g(y)=0 \\
& \mu^{T} g(y) \geqq 0 \\
& \lambda>0, \quad \mu \geqq 0 .
\end{aligned}
$$


Theorem 3.1. (Weak Duality). Let $x$ be feasible for $(P)$ and $(y, \lambda, \mu)$ be feasible for $(D)$. If $\lambda^{T} f$ is pseudoconvex and $\mu^{T} g$ is quasiconvex at $y$, then

$$
\lambda^{T} f(x) \geqq \lambda^{T} f(y)
$$

Proof: Since $g(x) \leqq 0$ and $\mu \geqq 0$,

$$
\mu^{T} g(x) \leqq 0 \leqq \mu^{T} g(y) .
$$

Using quasiconvexity of $\mu^{T} g$ at $y$, we get

$$
\mu^{T} \nabla g(y)(x-y) \leqq 0
$$

Therefore from equation (1)

$$
\lambda^{T} \nabla f(y)(x-y) \geqq 0 .
$$

But $\lambda^{T} f$ is pseudoconvex at $y$. Hence

$$
\lambda^{T} f(x) \geqq \lambda^{T} f(y)
$$

REMARK 1. It may be noted that the inequality (4) implies $f(x) \notin f(y)$.

THEOREM 3.2. Let $\bar{x}$ be feasible for $(P)$ and $(\bar{y}, \bar{\lambda}, \bar{\mu})$ be feasible for $(D)$ such that

$$
\bar{\lambda}^{T} f(\bar{x})=\bar{\lambda}^{T} f(\bar{y})
$$

If $\bar{\lambda}^{T} f$ is pseudoconvex and $\bar{\mu}^{T} g$ is quasiconvex at $\bar{y}$, then $\bar{x}$ is properly efficient for (P).

Proof: Let $x$ be any feasible solution for $(P)$. From the weak duality theorem,

$$
\bar{\lambda}^{T} f(x) \geqq \bar{\lambda}^{T} f(\bar{y}) .
$$

Using (5), we get

$$
\bar{\lambda}^{T} f(x) \geqq \bar{\lambda}^{T} f(\bar{x}) .
$$

Thus $\bar{x}$ is optimal for $\left(P_{\bar{\lambda}}\right)$. Hence by Lemma $1, \bar{x}$ is properly efficient for $(P)$.

THEOREM 3.3. Let $\bar{x}$ be feasible for $(P)$ and $(\bar{y}, \bar{\lambda}, \bar{\mu})$ be feasible for $(D)$ such that

$$
f(\bar{x})=f(\bar{y})
$$


If $\lambda^{T} f$ is pseudoconvex and $\mu^{T} g$ is quasiconvex at $y$ for each dual feasible $(y, \lambda, \mu)$, then $(\bar{y}, \bar{\lambda}, \bar{\mu})$ is properly efficient for the dual problem $(D)$.

PRoOF: First we show that $(\bar{y}, \bar{\lambda}, \bar{\mu})$ is efficient for $(D)$. Assume that it is not efficient, then there exists $\left(y^{*}, \lambda^{*}, \mu^{*}\right)$ feasible for (D) such that

$$
\begin{gathered}
f_{r}\left(y^{*}\right)>f_{r}(\bar{y}) \text { for some } r \in K \\
f_{i}\left(y^{*}\right) \geqq f_{i}(\bar{y}) \text { for all } i \in K_{r} .
\end{gathered}
$$

Therefore

or using (6),

$$
\begin{aligned}
& \lambda^{* T} f\left(y^{*}\right)>\lambda^{* T} f(\bar{y}) \\
& \lambda^{* T} f\left(y^{*}\right)>\lambda^{* T} f(\bar{x}),
\end{aligned}
$$

a contradiction to the weak duality theorem. Hence $(\bar{y}, \bar{\lambda}, \bar{\mu})$ is efficient for $(D)$. Assume now that it is not properly efficient. Then there exists a dual feasible solution $\left(y^{*}, \lambda^{*}, \mu^{*}\right)$ and an $i \in K$ such that $f_{i}\left(y^{*}\right)>f_{i}(\bar{y})$ and

$$
f_{i}\left(y^{*}\right)-f_{i}(\bar{y})>M\left(f_{j}(\bar{y})-f_{j}\left(y^{*}\right)\right)
$$

for all $M>0$ and all $j \in K_{i}$ satisfying $f_{j}(\bar{y})>f_{j}\left(y^{*}\right)$. This means that $f_{i}\left(y^{*}\right)-f_{i}(\bar{y})$ can be made arbitrary large whereas $f_{j}(\bar{y})-f_{j}\left(y^{*}\right)$ is finite for all $j \in K_{i}$. Therefore

or

$$
\begin{gathered}
\lambda_{i}^{*}\left(f_{i}\left(y^{*}\right)-f_{i}(\bar{y})\right)>\sum_{j \in K_{i}} \lambda_{j}^{*}\left(f_{j}(\bar{y})-f_{j}\left(y^{*}\right)\right) \\
\lambda^{* T} f\left(y^{*}\right)>\lambda^{* T} f(\bar{y}) .
\end{gathered}
$$

Using (6), we get

$$
\lambda^{* T} f\left(y^{*}\right)>\lambda^{* T} f(\bar{x}) .
$$

This again contradicts the weak duality theorem. Hence $(\bar{y}, \bar{\lambda}, \bar{\mu})$ is a properly efficient solution for $(D)$.

Theorem 3.4. (Strong Duality). Let $\bar{x}$ be a properly efficient solution of Problem $(P)$ and let $g$ satisfy the Kuhn-Tucker constraint qualification at $\bar{x}$. Then there exists $(\bar{\lambda}, \bar{\mu})$, such that $(\bar{y}=\bar{x}, \bar{\lambda}, \bar{\mu})$ is a feasible solution for $(D)$ and the objective values of $(P)$ and $(D)$ are equal. Also, if $\lambda^{T} f$ is pseudoconvex and $\mu^{T} g$ is quasiconvex at $y$ for every dual feasible solution $(y, \lambda, \mu)$, then $(\bar{x}, \bar{\lambda}, \bar{\mu})$ is a properly efficient solution for $(D)$.

ProOF: Since $\bar{x}$ is a properly efficient solution for $(P)$ at which the Kuhn-Tucker constraint qualification is satisfied, by Lemma 2 there exist $\bar{\lambda} \in R^{k}$ and $\bar{\mu} \in R^{m}$ such that

$$
\begin{array}{r}
\bar{\lambda}^{T} \nabla f(\bar{x})+\bar{\mu}^{T} \nabla g(\bar{x})=0 \\
\bar{\mu}^{T} g(\bar{x})=0 \\
\bar{\lambda}>0, \quad \bar{\mu} \geqq 0 .
\end{array}
$$


Therefore $(\bar{x}, \bar{\lambda}, \bar{\mu})$ is feasible for $(D)$. Also, the two vector objectives are equal. Hence, by Theorem $3.3,(\bar{x}, \bar{\lambda}, \bar{\mu})$ is a properly efficient solution for $(D)$.

THEOREM 3.5. (Converse Duality). Let $(\bar{y}, \bar{\lambda}, \bar{\mu})$ be a weak efficient solution for $(D)$, the $n \times n$ Hessian matrix $\nabla^{2}\left(\bar{\lambda}^{T} f(\bar{y})+\bar{\mu}^{T} g(\bar{y})\right)$ be positive or negative definite and $\nabla f_{i}(\bar{y}), i=1,2, \ldots, k$ be linearly independent. If $\lambda^{T} f$ is pseudoconvex and $\mu^{T} g$ is quasiconvex at $\bar{y}$, then $\bar{y}$ is a properly efficient solution for $(P)$.

Proof: Since $(\bar{y}, \bar{\lambda}, \bar{\mu})$ is a weak efficient solution for $(D)$, by Theorem 1 [2] there exist $\bar{u} \in R^{k}, \bar{v} \in R^{n}, \bar{w} \in R, \bar{\eta} \in R^{k}$ and $\bar{\nu} \in R^{m}$ such that

$$
\begin{aligned}
\bar{u}^{T} \nabla f(\bar{y})+\bar{v}^{T} \nabla^{2}\left(\bar{\lambda}^{T} f(\bar{y})+\bar{\mu}^{T} g(\bar{y})\right)+\bar{w} \bar{\mu}^{T} \nabla g(\bar{y}) & =0 \\
{[\nabla f(\bar{y})] \bar{v}+\bar{\eta} } & =0 \\
{[\nabla g(\bar{y})] \bar{v}+\bar{w} g(\bar{y})+\bar{\nu} } & =0 \\
\bar{w}\left[\bar{\mu}^{T} g(\bar{y})\right] & =0 \\
\bar{\eta}^{T} \bar{\lambda} & =0 \\
\bar{\nu}^{T} \bar{\mu} & =0 \\
(\bar{u}, \bar{w}, \bar{\eta}, \bar{\nu}) \geqq 0, \quad(\bar{u}, \bar{v}, \bar{w}, \bar{\eta}, \bar{\nu}) & \neq 0 .
\end{aligned}
$$

Now $\bar{\lambda}>0, \bar{\eta}^{T} \bar{\lambda}=0 \Rightarrow \bar{\eta}=0$. Therefore from (8)

$$
[\nabla f(\bar{y})] \bar{v}=0
$$

which with equation (1) gives

$$
\bar{\mu}^{T} \nabla g(\bar{y}) \bar{v}=0 .
$$

On multipling (7) by $\bar{v}$ from the right and using equations (14) and (15), we get

$$
\bar{v}^{T}\left[\nabla^{2}\left(\bar{\lambda}^{T} f(\bar{y})+\bar{\mu}^{T} g(\bar{y})\right)\right] \bar{v}=0 .
$$

Since $\nabla^{2}\left(\bar{\lambda}^{T} f(\bar{y})+\bar{\mu}^{T} g(\bar{y})\right)$ is assumed to be positive or negative definite, equation (16) gives $\bar{v}=0$.

Now suppose $\bar{w}=0$. Therefore from equation (9), $\bar{\nu}=0$. Also, equation (7) gives

$$
\bar{u}^{T} \nabla f(\bar{y})=0 .
$$

Since $\nabla f_{i}(\bar{y})$ are assumed to be linearly independent, (17) implies $\bar{u}=0$. Thus we get

$$
(\bar{u}, \bar{v}, \bar{w}, \bar{\eta}, \bar{\nu})=0
$$


a contradiction to (13). Hence $\bar{w}>0$.

Now from equation (9),

$$
g(\bar{y})=\frac{-\bar{\nu}}{\bar{w}} \leqq 0 .
$$

Therefore $\bar{y}$ is a feasible solution for $(P)$, and Theorem 3.2 implies that $\bar{y}$ is a properly efficient solution for $(P)$.

The following result gives sufficient conditions for a weak efficient solution of the dual problem $(D)$ to be properly efficient. It follows immediately from Theorems 3.3 and 3.5.

Corollary. Let $(\bar{y}, \bar{\lambda}, \bar{\mu})$ be a weak efficient solution for $(D)$ and let the hypotheses of Theorem 3.5 be satisfied. If $\lambda^{T} f$ is pseudoconvex and $\mu^{T} g$ is quasiconvex at $y$ for each dual feasible $(y, \lambda, \mu)$, then $(\bar{y}, \bar{\lambda}, \bar{\mu})$ is a properly efficient solution of the dual problem $(D)$.

REMARK 2. It may be noted that in Weir and Mond [10] the dual variable $\lambda \geq 0$. Therefore, their implication $w^{T} \lambda_{0}=0 \Rightarrow w=0$ in the converse duality theorem is erroneous.

Theorem 3.6. (Strict Converse Duality Theorem). Let $\bar{x}$ and $(\bar{y}, \bar{\lambda}, \bar{\mu})$ be feasible solutions for $(P)$ and $(D)$ respectively such that

$$
\bar{\lambda}^{T} f(\bar{x})=\bar{\lambda}^{T} f(\bar{y}) .
$$

If $\bar{\lambda}^{T} f$ is strictly pseudoconvex and $\bar{\mu}^{T} g$ is quasiconvex at $\bar{y}$, then $\bar{x}=\bar{y}$ and $\bar{y}$ is properly efficient for $(P)$.

Proof: We will assume $\bar{x} \neq \bar{y}$ and exhibit a contradiction.

Since $\bar{x}$ and $(\bar{y}, \bar{\lambda}, \bar{\mu})$ are feasible for $(P)$ and $(D)$ respectively, we have

$$
\bar{\mu}^{T} g(\bar{x}) \leqq 0 \leqq \bar{\mu}^{T} g(\bar{y}) .
$$

The quasiconvexity of $\bar{\mu}^{T} g$ at $\bar{y}$ implies

$$
\bar{\mu}^{T} \nabla g(\bar{y})(\bar{x}-\bar{y}) \leqq 0 .
$$

Equations (1) and (19) imply

$$
\bar{\lambda}^{T} \nabla f(\bar{y})(\bar{x}-\bar{y}) \geqq 0 .
$$

But $\bar{\lambda}^{T} f$ is strictly pseudoconvex at $\bar{y}$. Hence

$$
\bar{\lambda}^{T} f(\bar{x})>\bar{\lambda}^{T} f(\bar{y})
$$

a contradiction to (18). Hence $\bar{x}=\bar{y}$. Proper efficiency of $\bar{y}$ for $(P)$ now follows from Theorem 3.2. 


\section{Application}

We now apply our results to obtain a dual for the following nonlinear fractional vector minimum problem:

$$
\begin{aligned}
& \text { minimise } f(x)=\left[\frac{\phi_{1}(x)}{\psi(x)}, \frac{\phi_{2}(x)}{\psi(x)}, \ldots, \frac{\phi_{k}(x)}{\psi(x)}\right] \\
& \text { subject to } g(x) \leqq 0, \quad x \in S
\end{aligned}
$$

where

(i) $S \subseteq R^{n}$ is an open convex set,

(ii) $\phi: S \rightarrow R^{k}, g: S \rightarrow R^{m}$ are differentiable convex functions on $S$ and $\psi: S \rightarrow R$ is a differentiable concave function on $S$, and

(iii) $\phi(x) \geqq 0$ and $\psi(x)>0$ on $S$.

Therefore for each $\lambda>0, \lambda^{T} f$ is pseudoconvex and since $g$ is convex, convexity hypotheses in this paper are satisfied. Hence the dual problem $(D)$ becomes

$$
\begin{aligned}
& \text { maximise } f(y)=\left[\frac{\phi_{1}(y)}{\psi(y)}, \frac{\phi_{2}(y)}{\psi(y)}, \ldots, \frac{\phi_{k}(y)}{\psi(y)}\right] \\
& \text { subject to } \sum_{i=1}^{k} \lambda_{i}\left[\frac{\nabla \phi_{i}(y)}{\psi(y)}-\frac{\phi_{i}(y)}{\psi^{2}(y)} \nabla \psi(y)\right]+\mu^{T} \nabla g(y)=0 \\
& \mu^{T} g(y) \geqq 0, \quad \lambda>0, \mu \geqq 0, y \in S .
\end{aligned}
$$

On simplification, we get the following dual for $(F P)$ :

$$
\begin{aligned}
& \underset{\text { maximise }}{\text { subject to }} \\
& \qquad \begin{aligned}
& \lambda^{T} \nabla \phi(y)-\left(\sum_{i=1}^{k} v_{i}\right) \nabla \psi(y)+w^{T} \nabla g(y)=0 \\
& w^{T} g(y) \geqq 0 \\
& \lambda_{i} \phi_{i}(y)-v_{i} \psi(y)=0, \quad i=1,2, \ldots, k \\
& \lambda>0, v \geqq 0, w \geqq 0, y \in S
\end{aligned}
\end{aligned}
$$

where $\lambda, v \in R^{k}$ and $w \in R^{m}$.

In particular when $k=1$, we get the following pair of scalar nonlinear program and its dual 
Primal Problem:

$$
\begin{aligned}
& \text { minimise } \frac{\phi(x)}{\psi(x)} \\
& \text { subject to } g(x) \geqq 0, \quad x \in S
\end{aligned}
$$

Dual Problem:

$$
\begin{aligned}
& \text { maximise } f(y)=\frac{\phi(y)}{\psi(y)} \\
& \text { subject to } \\
& \nabla \phi(y)-v \nabla \psi(y)+w^{T} \nabla g(y)=0 \\
& w^{T} g(y) \geqq 0 \\
& \phi(y)-v \psi(y)=0 \\
& v, w \geqq 0, y \in S
\end{aligned}
$$

where $\phi: S \rightarrow R$ and $v \in R$. Other notations are as in $(F D)$.

REMARK 3. If $\psi$ is linear, then $\phi(x) \geqq 0$ is not required and the dual variable $v$ in the above dual problems is unrestricted in sign.

\section{REFERENCES}

[1] C.R. Bector, S. Chandra and M.V. Durgaprasad, 'Duality in pseudolinear multiobjective programming', Asia-Pacific J. Oper. Res. 5 (1988), 150-159.

[2] B.D. Craven, 'Lagrangian conditions and quasiduality', Bull. Austral. Math. Soc. 16 (1977), 325-339.

[3] A.M. Geoffrion, 'Proper efficiency and the theory of vector maximization', J. Math. Anal. Appl. 22 (1968), 618-630.

[4] O.L. Mangasarian, Nonlinear programming (McGraw-Hill, New York, 1969).

[5] B. Mond and T. Weir, 'Generalized concavity and duality', in Generalized concavity in optimization and economics, Editors S. Schaible and W.T. Ziemba, pp. 263-279 (Academic Press, New York, 1981).

[6] C. Singh, 'Optimality conditions in multiobjective differentiable programming', J. Optim. Theory Appl. 53 (1987), 115-123.

[7] C. Singh, 'Duality theory in multiobjective differentiable programming', J. Inform. Optim. Sci. 9 (1988), No. 2, 231-240.

[8] T. Weir, 'Proper efficiency and duality for vector valued optimization problems', J. Austral. Math. Soc., Series A 43 (1987), 21-34.

[9] T. Weir and V. Jeyakumar, 'A class of nonconvex functions and mathematical programming', Bull. Austral. Math. Soc. 38 (1988), 177-189. 
[10] T. Weir and B. Mond, 'Generalized convexity and duality in multiple objective programming', Bull. Austral. Math. Soc. 39 (1989), 287-299.

\author{
Department of Mathematics \\ University of Roorkee \\ Roorkee \\ India 247667
}

\author{
Department of Mathematics \\ University of Alexandria \\ Egypt
}

